\title{
AGRICULTURA ECOLÓGICA Y COOPERATIVISMO EN ANDALUCÍA Una fórmula de desarrollo rural alternativa
}

\author{
Alberto del Campo Tejedor \\ Departamento de Trabajo Social \\ Universidad Pablo de Olavide (Sevilla) \\ Javier Navarro Luna \\ Departamento de Geografía Física y Análisis Geográfico Regional \\ Universidad de Sevilla
}

\section{RESUMEN}

La agricultura ecológica es un sistema de producción que aspira a compaginar el respeto al medio ambiente, la producción de alimentos de calidad sin el uso de sustancias químicas de síntesis, y una mayor rentabilidad que la producción agraria convencional. El modelo agroecológico, especialmente en aquellas iniciativas gestionadas bajo formas de economía social (sociedades cooperativas), se afianza en Andalucía como una eficaz alternativa a la desestructuración y desarticulación del panorama agrario andaluz. A través de un estudio de caso en la comarca de Los Pedroches (Córdoba), este texto analiza las causas del crecimiento de este sistema productivo, así como los principales obstáculos para su consolidación.

Palabras clave: Andalucía. Agricultura ecológica. Cooperativismo. Desarrollo rural.

\begin{abstract}
A formula to an alternative rural development. The organic farming is a production system that aspires to reconcile the respect for the environment, the production of high quality food without using chemical substances, and a bigger rentability than conventional farming. The agroecological model, specially in those enterprises managed under forms of social economy (cooperatives), is becoming strong in Andalusia as an efficient alternative to the destructuration and the dislocation of the andalusian agricultural wealth. With a case study in the region of Los Pedroches (Córdoba, Spain), this text analyzes the reasons for the increase of this productive system, as well as the principal handicaps for its consolidation.
\end{abstract}

Key words: Andalusia. Organic farming. Cooperativism. Rural development. 


\section{Introducción}

El progresivo deterioro de las condiciones socioeconómicas y de las perspectivas de desarrollo en el mundo rural no ha restado importancia a la actividad agraria en Andalucía. Aún es considerada como un pilar de nuestra economía, tanto por los recursos generados ${ }^{1}$ como por el empleo que sustenta ${ }^{2}$. Es cierto que ha sufrido una radical transformación en las últimas tres décadas pero la rápida capitalización, los avances en las técnicas de producción y la progresiva modernización del sector no han impulsado paralelamente un proceso de desarrollo con creación de empleo, aumento de las rentas, articulación de la población rural y reequilibrio de las desigualdades intrarregionales. Bien al contrario, el tejido agrario de Andalucía sigue inmerso en una grave desarticulación, marcado por la concentración en una agricultura extensiva especializada en monocultivos y en el suministro de materias primas agrícolas. Este fenómeno, unido al desmantelamiento de muchas de las actividades tradicionales del campo, así como a la ausencia de actividades productivas alternativas, hacen del campo andaluz uno de los sectores más desestructurados e invertebrados de nuestra economía.

No obstante, a pesar de este desolador panorama, algunas comarcas andaluzas están llevando a cabo en los últimos diez años audaces experiencias socioeconómicas que afianzan nuevos procesos de producción agraria que hace sólo una década permanecían en estado embrionario. Así, por ejemplo, encontramos iniciativas de intensificación agrícola en las zonas del litoral especializadas en cultivos extratempranos de alto rendimiento, colonización agraria de tierras poco productivas con sistemas de invernaderos, mecanización y modernización de las fincas con grandes sumas de inversión y probada rentabilidad como el sistema de goteo en olivares. En algunos casos constituyen iniciativas empresariales aisladas con capacidad posterior de arrastre, mientras que la mayoría está impulsada por el enfoque vigente de desarrollo rural, amparado en las cuantiosas ayudas procedentes de las arcas europeas. Un cambio, por tanto, de carácter institucional con el que se pretende inducir una diversificación productiva en el medio rural (turismo, ocio, agricultura ecológica, artesanía, ...) que contribuya a frenar el declive estructural en las comarcas de economía de base agraria. Así en la última década en Andalucía han surgido agrupaciones territoriales de municipios, auténticas comarcas de desarrollo rural, acogidas al caudal de fondos europeos procedentes de programas como el LEADER II y el PRODER. Dentro de este proceso de búsqueda de alternativas a la crisis del campo se encuentra uno de los sistemas de producción agraria que más ha crecido en los siete últimos años en Andalucía: la agricultura ecológica.

\section{La agricultura ecológica en España y Andalucía}

Con este término se define un sistema agrario cuyo objetivo fundamental es la obtención de alimentos de máxima calidad respetando el medio ambiente y conservando o incrementando la fertilidad de la tierra, mediante la utilización óptima de los recursos naturales y sin el empleo de productos químicos de síntesis. Como han destacado diversos estu-

1 La producción final agraria alcanzó en Andalucía, en 1999, un valor de 1.162.939 millones de ptas., de los que 922.563 correspondieron al subsector agrícola.

2 Con una media del $12 \%$ de la población ocupada, y que alcanza más del $50 \%$ en comarcas de montaña y del interior andaluz. 
dios (Reijntjes et al., 1992; Colmenares et al., 1994; Lampkin, 1998) no se trata de una vuelta bucólica y romántica al agrarismo tradicional sino de un planteamiento integrador que aprovecha técnicas y prácticas antiguas y experimenta con moderna tecnología para un manejo de los recursos naturales que no hipoteque la continuidad productiva ni erosione el patrimonio medioambiental.

Este modo de enfocar el agro, que hunde sus raíces históricas en la centroeuropa de los años veinte ${ }^{3}$ y que se desarrolla en contraposición al modelo convencional —productivista, industrial, químico - ha permanecido hasta hace pocos años con una productividad puramente testimonial, lo que ha provocado una imagen estereotipada de la agricultura ecológica como modelo utópico e insostenible. De hecho su desarrollo en España ha sido relativamente tardío, en relación al resto de países europeos, pero espectacular, en especial desde mediados de la década de los noventa. Según los registros del C.R.A.E (Consejo Regulador de Agricultura Ecológica), en 1991 tan sólo 346 agricultores cultivaban, generalmente en pequeños huertos familiares, poco más de 4.000 hectáreas en toda España, una cifra testimonial en relación a las 55.000 que 2.600 franceses cultivaban en 1987. Sin embargo ya en 1995 la cifra de agricultores ecológicos en España se había multiplicado por

Cuadro 1

$N^{\circ}$ DE PRODUCTORES Y SUPERFICIES EN AGRICULTURA ECOLÓGICA EN 1999

\begin{tabular}{|l|c|c|}
\hline Comunidad Autónoma & Productores & Superficie total (ha) \\
\hline Andalucía & 2.489 & $62.318,13$ \\
Aragón & 167 & $15.638,42$ \\
Asturias & 25 & 64,75 \\
Islas Baleares & 117 & $3.623,40$ \\
Canarias & 269 & $5.075,40$ \\
Cantabria & 61 & 842,05 \\
Castilla-La Mancha & 159 & $7.632,12$ \\
Castilla y León & 191 & $43.245,34$ \\
Cataluña & 318 & $9.570,00$ \\
Extremadura & 6.743 & $167.833,31$ \\
Galicia & 67 & 181,85 \\
Madrid & 42 & $1.303,60$ \\
Región de Murcia & 324 & $8.406,60$ \\
C.F. de Navarra & 163 & $6.793,10$ \\
La Rioja & 52 & $1.342,00$ \\
País Vasco & 52 & 347,19 \\
C.Valenciana & 573 & $17.947,00$ \\
Total Nacional & 11.812 & $352.164,26$ \\
\hline
\end{tabular}

FUENTE: Hechos y Cifras del sector agroalimentario Español 2000.

3 A principios de los años 20 el austriaco Rudolf Steiner (1860-1925) aplicó en unos cursos para agricultores en Koberwitz (Silesia) los postulados de la llamada antroposofía, teoría filosófica antimaterialista, a la agricultura, sentando las bases de la Agricultura Biodinámica. Poco después, un reducido número de simpatizantes de Steiner llevó a cabo las técnicas de cultivo formuladas por éste, logrando comercializar en 1928 los primeros alimentos bajo una etiqueta de calidad con el nombre de la diosa griega Demeter. 
tres (de 356 a 1.042) y la superficie por seis, acercándose a las 25.000 hectáreas. Desde entonces los datos no han hecho más que apuntalar el enorme crecimiento y expansión de la agricultura ecológica.

El papel jugado por Andalucía ha sido primordial en el rápido crecimiento de este sistema de producción, de tal forma que hasta 1997 se sitúa al frente de la producción nacional, tanto en superficie cultivada como en el número de agricultores involucrados. Desde 1998 el relevo lo toma Extremadura con crecimientos extraordinarios, que la sitúan a la cabeza de España en número de productores y extensión de cultivos, muy por encima del resto. Ambas junto con Castilla y León polarizan la mayor extensión de tierras de cultivo (dos terceras partes del total) y aglutinan, las dos primeras, el $80 \%$ de los productores. Mientras en el resto de comunidades la incidencia de este sistema agrario se presenta de forma muy desigual (cuadro $\mathrm{n}^{\circ} 1$ ).

La agricultura ecológica en Andalucía ha seguido similares pautas de desarrollo que en otras zonas de nuestro país, acentuando su crecimiento a partir de 1995 y 1996. En 1992 tan sólo 2.212 hectáreas eran cultivadas ecológicamente, pero tres años más tarde, en 1995, la superficie se había triplicado, alcanzando las 6.455 has. cultivadas por 277 agricultores. Mil novecientos noventa y seis supondrá el despegue definitivo de la agricultura ecológica en Andalucía: más de 500 agricultores deciden abandonar los métodos convencionales y adaptar sus sistemas de producción con métodos ecológicos. Con más de 20.000 hectáreas, Andalucía se convierte en el punto de referencia para el resto. A finales de 1997 los productores ecológicos superan el millar, con explotaciones que suman un total de 32.497 has. El crecimiento, por tanto, se dispara: en 1998 el número de agricultores asciende a 1.769 (incremento del 639\% en cuatro años) y la superficie cultivada supera las 47.000 hectáreas. A principios del año 2000 son ya 2.489 productores ecológicos que cultivan 62.318 has. en las ocho provincias andaluzas, lo que supone un aumento en ocho años del $2.717 \%$ (cuadro $\mathrm{n}^{\mathrm{o}} 2$ ), y al igual que a nivel nacional aquí se constata disparidades provinciales en la extensión y utilización de estos sistemas agrarios, con un protagonismo de Córdoba y, en menor medida Granada y Almería.

Cuadro 2

SUPERFICIE Y NÚMERO DE PRODUCTORES ECOLÓGICOS EN ANDALUCÍA EN EL 2000

\begin{tabular}{|l|c|c|c|}
\hline Provincia & Productores & $\begin{array}{c}\text { Superficie } \\
\text { (Has.) }\end{array}$ & $\begin{array}{c}\text { Superficie } \\
\text { ( \% })\end{array}$ \\
\hline Córdoba & 917 & $21.544,7$ & 34,5 \\
Granada & 249 & $10.532,4$ & 16,9 \\
Almería & 432 & 9.936 & 15,9 \\
Sevilla & 153 & $6.701,2$ & 10,7 \\
Málaga & 365 & $4.378,8$ & 7,0 \\
Huelva & 129 & $3.587,8$ & 5,7 \\
Jaén & 180 & $2.921,6$ & 4,6 \\
Cádiz & 64 & $2.715,2$ & 4,3 \\
Andalucía & 2.489 & $62.318,1$ & 100,0 \\
\hline
\end{tabular}

FUENTE: C.A.A.E.,2000. 
La relevancia socioeconómica de la agricultura ecológica en Andalucía incide de forma directa, sin embargo, en un número mucho mayor que esas 2.489 personas, y ello por las siguientes razones:

1. El número de agricultores que aparecen inscritos (2.489) refiere sólo a los titulares de las fincas, independientemente de que en ellas trabajen otros miembros de la unidad familiar.

2. Muchos agricultores, como los propietarios de grandes extensiones de olivar o frutales, son una fuente de trabajo para muchas familias, a las que contratan, bien en época de recolección, bien durante todo el año. Así, algunos latifundistas de olivar en Baena cuentan, según la época del año, hasta con 150 operarios, que no figuran a nivel estadístico.

3. En los registros del C.A.A.E. (órgano de certificación y control autonómico de Andalucía), el número de agricultores es asignado a cada cultivo en función de su actividad principal. Aquellos productores que, como es frecuente, se dedican a varios cultivos distintos a la vez, sólo figuran estadísticamente en aquel que les reporta mayores ingresos.

Por todo ello, si bien el número de hectáreas que figura en las estadísticas (hoy 62.318) sí obedece a la superficie total explotada en los distintos cultivos, la cifra de los productores no refleja la cantidad de personas que dependen hoy económicamente de la agricultura ecológica.

Las explotaciones ecológicas andaluzas muestran una superficie media (26,8 has. por agricultor) superior a la de las fincas convencionales (17,9 has. por agricultor) debido fundamentalmente a las grandes extensiones de olivar y dehesa. El olivar, con algo más de 21.000 has., explotadas por 1.205 agricultores como actividad principal, es con diferencia el cultivo más importante de Andalucía, localizado mayoritariamente en Córdoba (65\%) . La superficie de dehesa supera las 11.000 has., de nuevo centradas en Córdoba y Sevilla que en conjunto suman las tres cuartas partes del total. Estas grandes extensiones sólo son utilizadas por poco más de un centenar de ganaderos ecológicos. La misma infraexplotación la encontramos en las más de 8.000 has. de bosque y monte, en las que otro centenar

Cuadro 3

MAGNITUDES DE LAS EXPLOTACIONES ECOLÓGICAS

\begin{tabular}{|l|c|r|c|c|c|}
\hline & Hectáreas & $\boldsymbol{( \% )}$ & Agricultores & $\boldsymbol{( \% )}$ & $\begin{array}{c}\text { Media } \\
\text { Superficie/Explotación }\end{array}$ \\
\hline$<5$ Has. & 2.822 & 5,9 & 732 & 41,3 & 3,8 \\
$10-25$ Has. & 11.010 & 23,2 & 572 & 32,3 & 19,2 \\
$25-50$ Has. & 12.204 & 25,7 & 267 & 15,1 & 45,7 \\
$50-100$ Has. & 10.232 & 21,5 & 138 & 7,8 & 74,1 \\
100-300Has. & 9.150 & 19,2 & 55 & 3,1 & 166,3 \\
$>$ 300 Has. & 2.052 & 4,2 & 5 & 0,2 & 410,4 \\
\hline TOTAL & 47.470 & 100 & 1.769 & 100 & 26,8 \\
\hline
\end{tabular}

FUENTE: C.A.A.E., 2000. 
de agricultores practica la recolección silvestre. Mayor importancia tienen los cultivos de frutales de secano (10.297 has.) y de herbáceos de secano (8.157 has.) explotados respectivamente por 634 y 187 agricultores. El resto de cultivos no supera ninguno el centenar de productores, si los catalogamos en función de su actividad principal (aunque hay que recordar que en muchos casos en las explotaciones se realizan varios tipos de cultivo): hortícolas (790 has.), cítricos (529 has.), frutas subtropicales (333 has.), plantas aromáticas y medicinales (280 has.), herbáceos de regadío (263 has.), frutales de regadío (181 has.), viñedos (161 has.), cultivos bajo invernaderos (23 has.), viveros (7 has.) y plantaciones pratenses (1 ha.).

Cuadro 4

SUPERFICIE DE CULTIVO ECOLÓGICO EN ANDALUCÍA (HAS.)

\begin{tabular}{|l|c|c|c|c|c|c|c|c|c|}
\hline & Almería & Cádiz & Córdoba & Granada & Huelva & Jaén & Málaga & Sevilla & $\begin{array}{c}\text { Superficie } \\
\text { total }\end{array}$ \\
\hline Apicultura & - & - & 351,2 & - & - & - & - & - & 351,2 \\
Aromáticas & 274,2 & - & - & - & - & - & 1,9 & 4,6 & 280,7 \\
Bosque & 854,3 & 268,4 & $1.347,6$ & $4.776,5$ & 464,2 & 102,7 & 311,9 & 82,5 & $8.208,4$ \\
Cítricos & 159,2 & 3,7 & 1,3 & 1,7 & 32 & - & 193,4 & 137,2 & 529 \\
Dehesa & 44,3 & 327,9 & $4.846,1$ & $1.224,1$ & $1.239,7$ & 366,9 & 343,5 & $3.068,5$ & $11.461,4$ \\
Frutales regadío & 81,6 & 3,5 & 12,2 & 43 & 4,1 & 0,3 & 30,5 & 5,9 & 181,6 \\
Frutales secano5 & 190,2 & - & 16,4 & $1.790,1$ & $1.209,6$ & 6,3 & 2.078 & 6,3 & $10.297,1$ \\
Herbáceo regadío & 43,9 & 21,1 & 21,7 & 70,5 & - & 8,7 & 22 & 75,3 & 263,4 \\
Herbáceo secano & $2.763,8$ & $1.681,4$ & $1.091,8$ & 1.915 & 50,7 & 53,1 & 270,3 & 330,8 & $8.157,1$ \\
Hortícolas & 188,3 & 117,8 & 12 & 62,8 & 140,6 & 10,3 & 174,3 & 84 & 790,4 \\
Invernadero & 7 & 1,9 & - & 4,4 & 3,5 & - & 6,5 & - & 23,4 \\
Olivar & 283,6 & 284,6 & 13.790 .1 & 596,2 & 401,7 & $2.372,9$ & 638,3 & $2.901,9$ & $21.269,5$ \\
Pratenses & - & - & 0,8 & 0,7 & - & - & - & - & 1,6 \\
Subtropicales & - & 4,5 & - & 6,6 & 32 & - & 290,6 & - & 333,7 \\
Viña & 43,5 & - & 52,9 & 39 & 9,5 & 0,03 & 16,2 & 0,2 & 161,5 \\
Vivero & 43,5 & - & 1,3 & $0,2-$ & 1,2 & - & 1 & 3,6 & 7,5 \\
\hline Superficie total & 9.936 & $2.715,2$ & $21.544,7$ & $10.532,4$ & 3.587, & $82.921,6$ & $4.378,8$ & $6.701,2$ & $62.318,1$ \\
\hline
\end{tabular}

FUENTE: C.A.A.E. 2000.

\subsection{Razones del crecimiento.}

Es difícil precisar los factores que más han contribuido al auge de la agricultura ecológica. En ocasiones se ha señalado la progresiva degradación de la naturaleza como el fundamental factor desencadenante de la avalancha de conversiones hacia una agricultura más respetuosa con el medio, junto a la necesaria creación de un mercado que demanda ese tipo de productos. También se alude a iniciativas locales que han extendido y generalizado esos sistemas agrarios, especialmente localizadas en áreas marginales y deprimidas. Sería, en definitiva, el resultado de las profundas transformaciones vividas por el campo en pasadas 
épocas, en los cambios de las funciones económicas en las áreas rurales. En respuesta a ese nuevo contexto las instituciones públicas —nacionales y comunitarias- han asumido esas transformaciones mediante el giro de sus políticas, desde posiciones agrarias a rurales, apostando por un nuevo enfoque en el que sobre aspectos productivos priman los medioambientales, sociales y culturales ${ }^{4}$.

En un ámbito más global, y haciendo un esfuerzo de abstracción y compresión, cabe citar los siguientes factores como cooperadores de la consolidación de la agricultura ecológica en Andalucía:

- Condiciones edafológicas, climatológicas, geográficas, culturales y medioambientales idóneas para muchos cultivos ecológicos de consolidada demanda (olivo, subtropicales, cítricos).

- Menor impacto medioambiental de los modelos desarrollistas debido a una tardía aplicación de los modos de producción más nocivos, así como un uso menos agresivo, continuado y generalizado de dicho modelo en muchas comarcas andaluzas, lo que facilita la labor de reconversión.

- Rica tradición agraria con multitud de variedades autóctonas, muy apreciadas por el consumidor, y un notable corpus de conocimiento tradicional en cuanto a prácticas y técnicas de cultivo sostenibles.

- Creciente sensibilización ecologista a nivel popular que defiende la protección del medio no sólo en foros académicos sino también a través de movimientos sociales como organizaciones no gubernamentales, asociaciones de productores y consumidores, sindicatos, y movimientos jornaleros.

- Aumento considerable de la demanda del mercado externo, especialmente de centroeuropa y progresiva apertura de otros mercados como el asiático y el norteamericano.

- Sobreprecios, tanto a nivel de comercio andaluz como en el mercado exterior, que llegan a duplicar el valor de los alimentos producidos convencionalmente.

- Consolidación y aumento de la productividad de muchas fincas después de varios años de cultivos ecológicos, debido a los efectos favorables sobre el agro a medio y largo plazo: aumento de la fertilidad del suelo, independencia de insumos externos, aprendizaje de estrategias adaptativas, etc.

- Éxito de fórmulas empresariales alternativas como las Sociedades de Cooperativas, y las federaciones de asociaciones.

- Paulatino interés en la creación de cadenas de transformación, elaboración, empaquetado y embotellado de los alimentos bajo marcas propias.

4 Resulta necesario indicar en este sentido la aprobación por la Comisión Europea del Programa Medioambiental 2000/06, dentro del Programa de Ayuda al Desarrollo Rural (Reglamento CE 1257/99), Programa que en su conjunto presenta unos principios de buenas prácticas agrarias dirigidas tanto a la utilización de sistemas no agresivos con el medioambiente (agricultura y ganadería ecológicas), lucha contra la erosión, protección de flora y fauna en humedales, extensificación de la producción agraria (práctica del barbecho tradicional, entre otras) etc. El territorio de aplicación de las ayudas se extiende por toda España, ya que además de las ya reconocidas con anterioridad (Parques Nacionales, Ramsar, ZEPAS y zonas específicas por su problemática medioambiental y agronómica), se incluirán todas aquellas zonas susceptibles de incorporar ayudas al desarrollo de prácticas agrarias sostenibles y respetuosas con los valores naturales. 
- Apoyo financiero de las Administraciones (europeas, nacionales y autonómicas) en forma de subvenciones a la reconversión, aprobación de investigaciones, colaboración en jornadas y congresos, etc.

- Labor de investigación en determinados departamentos universitarios, centros de investigación y experimentación y otras asociaciones y organizaciones convervacionistas, políticas y agrarias.

- Difusión y promoción de los productos ecológicos andaluces en ferias, congresos, jornadas, tanto internacionales como autonómicas.

— Institucionalización de la agricultura ecológica a través órganos de control y certificación.

A pesar de estas favorables variables y de buenas perspectivas de futuro para el sector agroecológico, existen aún muchos factores limitantes que obstaculizan un mayor desarrollo, además de los problemas estructurales de la agricultura andaluza convencional (desarticulación territorial, éxodo rural, cambio generacional, baja capitalización, etc.), muchos de los cuales afectan en igual medida a la agricultura ecológica.

\subsection{Los frenos al crecimiento.}

Las investigaciones que estamos llevando a cabo desvelan importantes cambios en la impregnación y significación de la agricultura ecológica en Andalucía pero también han visto confirmadas lamentablemente algunas de las conclusiones negativas que ya apuntaran otros estudios anteriores (DOXA, 1991; Ecomarket, 1993; Cánovas et al., 1993; CEPA, 1993; Alonso y Guzmán, 1993; RAEA, 1994; Cénit et al., 1996; Gutiérrez y Albi, 1996; Del Campo, 2000') en cuanto a las limitaciones de esta actividad.

En términos generales, la problemática de la agricultura ecológica estriba en que ésta supone, en mayor o en menor medida, un cambio en los planteamientos productivos, las técnicas y prácticas de cultivo, las vías de comercialización e incluso la cultura del trabajo, con respecto a la agricultura convencional. Así mientras que ésta aborda separadamente cada uno de los aspectos del proceso productivo (mejora genética, adaptación de variedades, fertilización química, lucha contra las adventicias con pesticidas, etc.), la agricultura ecológica exige para el éxito una visión sistémica, integral, holística y multidependiente, que incluya un manejo del suelo a largo plazo, el reciclaje de desechos, el aumento de la biodiversidad y el escrupuloso respeto a la legislación medioambiental y ecoagraria. Junto a esta dificultad genérica, atenuada a medida que el agricultor se acostumbra al nuevo modelo productivo, siguen existiendo barreras de todo orden que dificultan el éxito de muchas explotaciones:

- Escaso nivel de formación e información, por lo que el agricultor se ve obligado a rebuscar antiguas prácticas agrarias ocultas en el saber popular o a experimentar in situ con procedimientos que ignora.

5 La propia Unión Europea reconoce su extensión como sistema agrario en su territorio, aunque también asume algunos de los problemas existentes, como la falta de salidas comerciales y la necesidad de un mayor esfuerzo en materia de transformación y comercialización de los productos. 
- Limitada y dispersa oferta de productos fitosanitarios y fertilizantes por las casas comerciales lo que plantea graves problemas de abastecimiento en algunas zonas distantes de los centros de actividad comercial.

- Elevados costes unitarios de producción, especialmente en los primeros años, debido a la inexperiencia del agricultor, que junto a los menores rendimientos de las explotaciones, encarece los alimentos.

- Distribución irregular e intermitente que dificulta un aprovisionamiento continuado y de calidad homogénea, junto a una escasa oferta de productos ecológicos en los lugares habituales de compra.

- Bajo nivel de conocimiento de las propiedades de la producción ecológica e incluso de su mera existencia, relacionado con la escasa cultura y educación agroambiental y la ausencia de campañas de comunicación y publicidad.

- Competencia desleal y fraudulenta de productos convencionales que se comercializan como biológicos, ecológicos o naturales.

\section{El asociacionismo agrario. Una respuesta local al declive productivo del mundo rural}

La creciente demanda de alimentos producidos de forma ecológica origina en los agricultores andaluces la dificultad de abastecer de forma continuada y uniforme a comercializadoras y consumidores que solicitan cada vez mayor homogeneidad, disponibilidad y regularidad en el suministro. Muchos de ellos, pequeños productores, sin capacidad de cultivar, transformar y comercializar sus alimentos durante todo el año, se están agrupando bajo la forma de Sociedades Cooperativas. Esta fórmula, inserta dentro de lo que se ha dado en denominar Economía Social, actualmente en expansión y en la que España ocupa un lugar destacado (Holstrom, 1994), está siendo una vía útil para el desarrollo de estructuras industriales en el sector agrario ecológico.

Asistimos en la actualidad, en el mundo rural, a un doble proceso en el fenómeno del asociacionismo. De una parte los correspondientes al impulso institucional a través de la proliferación de mancomunidades y consorcios, cuya finalidad principal, en su origen, y dentro del ámbito estrictamente rural, era la de asumir unos servicios básicos que los pequeños municipios integrantes, la mayoría se veían incapaces de ofrecer por si solos. La puesta en práctica de estas fórmulas asociativas, desde la aprobación de la Ley de Bases de Régimen Local en 1985, así como de las leyes autonómicas desarrolladas ${ }^{6}$, ha permitido un crecimiento continuo de su número, del volumen de población rural asistida, de su extensión territorial y del incremento en la prestación de servicios. Su éxito ha sido indudable y su experiencia en comarcas con caracteres homogéneos ha servido, en muchos casos, para constituir grupos de acción local sujetos a las iniciativas comunitarias ${ }^{7}$ LEADER y PRODER, que acogen un total de 662 municipios, con una extensión de $77.219 \mathrm{Km}^{2}$ y una población de 3.018.712 habitantes. De otra parte asistimos a la agrupación de pequeños o

6 En principio corresponde tan sólo a las CC.AA. de la vía 151, así como Navarra, Valencia y Canarias. Mientras el resto tan sólo puede hacerlo mediante la reforma de sus Estatutos (COSCULLUELA, 1993).

7 En Andalucía la práctica totalidad de municipios rurales, hasta 662, se encuadran en estos dos programas. Los ubicados en las áreas más desfavorecidas y en declive, que se corresponde con las zonas montañosas, se integran en el LEADER en un total de 22 grupos, mientras que al PRODER se acogen 27 del resto de comarcas rurales. 
medianos productores en cooperativas, sociedades agrarias de transformación, organizaciones de productores agrarios o fórmulas similares. El auge de este tipo de asociacionismo, no circunscrito sólo a comarcas agrarias deprimidas sino extendido en su conjunto, registra en España cifras significativas. Si tomamos valores de facturación se alcanza el $40 \%$ de la producción final agraria, con un marcado protagonismo de ciertas producciones agrarias, donde las cooperativas suman más de la mitad de la producción: Tabaco (100\%); arroz (80\%); vino y aceite (70\%); cítricos $(50 \%)$. En este contexto Andalucía presenta una situación brillante, ya que una de cada tres cooperativas ${ }^{8}$ radica en esta comunidad ${ }^{9}$, para un total nacional de 4.426 .

Desde la perspectiva de la organización, estas agrupaciones siguen un modelo sociolaboral en el que la relación capital/trabajo toma una configuración específica: los trabajadores son al mismo tiempo los propietarios de la empresa. Esta situación presenta múltiples ventajas; unas de corte social (incremento del empleo y aumento de las expectativas laborales), otras de corte económico (viabilidad del proyecto, competitividad de las producciones...) pero, sobre todo, una de indudable trascendencia territorial, como es la condición local de estos procesos asociativos que junto al marcado carácter endógeno de sus producciones, les permite fijar estrategias de desarrollo a largo plazo. Con ello se consigue alcanzar la viabilidad de la actividad, fomentar la competitividad no mediante el productivismo sino con la busqueda de calidad, la diversificación hacia otras áreas de actividad no meramente agrarias (artesanía, ocio ...). En definitiva, estas empresas pueden configurar sistemas cercanos a la que algunos han denominado «multifuncionalidad de la agricultura» (Givord, 2000) y que es, en definitiva, el modelo rural europeo.

Si el fenómeno cooperativista está en un proceso de expansión en el conjunto de la producción agraria, también ocurre de igual forma con la agricultura ecológica. Estudios empíricos inciden en la motivación sociotrópica (sociotropic factors), es decir el considerar el

Cuadro 5

COOPERATIVAS, SOCIOS Y FACTURACIÓN DE LOS PRINCIPALES SECTORES AGRARIOS ESPAÑOLES

\begin{tabular}{|l|c|c|c|}
\hline Sector & $\mathbf{N}^{\mathbf{0}}$ Cooperativas & $\mathbf{N}^{\mathbf{0}}$ Socios & $\begin{array}{c}\text { Facturación } \\
\text { (millones ptas.) }\end{array}$ \\
\hline Suministros & 1.890 & 458.000 & 408.930 \\
Frutas y Hortalizas & 989 & 165.000 & 377.959 \\
Aceite de oliva & 950 & 295.000 & 216.000 \\
Vitivinícola & 753 & 160.000 & 107.043 \\
Cultivos herbáceos & 705 & 120.000 & 120.274 \\
Ganadería & 602 & 80.000 & 362.954 \\
Cultivos industriales & 95 & 18.500 & 72.164 \\
\hline
\end{tabular}

FUENTE: Hechos y Cifras del sector agroalimentario Español 2000.

8 Ministerio de Trabajo y Asuntos Sociales, año 2000.

9 Comunidad Valenciana (11,47\%), Castilla y León (11,07\%) y Castilla-La Mancha $(9,64 \%)$ ocupan los puestos siguientes. Mientras que en los niveles inferiores y con cifras muy raquíticas se sitúan, Cantabria (con tan sólo 3 agrupaciones); Islas Baleares (con 19) y Asturias (con 23). 
problema ambiental como un problema colectivo de interés general, lo que explicaría el aumento de acciones colectivas en defensa del medio (Rohrschneider, 1988). Podríamos pensar que, en el caso de los numerosos cooperativistas ecológicos andaluces, el interés crematístico propio y la motivación colectivista sociotrópica son compatibles e indisociables, como ha observado Rohrschneider (1988) para casos similares:

«los motivos de interés propio constituyen una fuerza importante en el ámbito económico de la política, pero la aparición de temas no económicos en las naciones industriales avanzadas (el problema ecológico, por ejemplo), puede disminuir el valor explicativo de estos motivos».

Una de las razones de la rápida asimilación cultural del modelo agroecológico se debe a que las prácticas y los manejos del suelo, la forma de organización empresarial, y los valores que sustentan este enfoque permiten y fomentan la imbricación de las culturas agrarias locales con los modernos avances tecnológicos. Muchas de estas cooperativas, nacidas no por casualidad en ámbitos alejados del mundo urbano, están conjugando ambas esferas, y hacen realidad el desideratum que formulara Víctor Toledo (1992), uno de los padres de la agroecología:

«la agricultura ecológica no intenta un romántico e inviable retorno a las formas preindustriales de producción; lo que busca es implementar una estrategia que modernice el agro a partir de un manejo adecuado de la naturaleza y del reconocimiento, —no de la destrucción—, de la tradición rural».

En Andalucía estos colectivos se distribuyen por todo el territorio, pero existen determinados enclaves donde han proliferado con mayor arraigo (el Valle del Guadalhorce malagueño, el Valle de los Pedroches cordobés, la Sierra de Segura en Jaén). Igualmente el número de socios de las cooperativas varía enormemente desde la media docena de pequeños horticultores de huerto hasta varios centenares de olivareros. Las cooperativas de agricultores ecológicos en Andalucía suelen dedicar su producción mayoritariamente a la exportación (se calcula que en un $90 \%$ ), aprovechando los altos precios que pagan los consumidores centroeuropeos por unos alimentos libres de productos químicos, aunque también establecen relaciones comerciales a través de canales de distribución nacionales, autonómicos e incluso comarcales, manteniendo en la mayoría de los casos la venta directa en la propia industria. A pesar de que gran parte de la producción se vende a granel, muchos de estos cooperativistas han optado por invertir en cadenas de elaboración (empaquetado, embotellado, etc.) para comercializar sus productos bajo una marca propia. Es el caso, por ejemplo, de los olivareros de la comarca de los Pedroches o de la Sierra de Segura que venden su preciado aceite virgen bajo las marcas Olivalle y Oro de Génave, respectivamente. Cultivar y producir para una marca propia tiene indudables ventajas económicas y sociales: obtención de un mayor valor añadido al producto, en el primer caso, y un mayor esfuerzo conjunto entre todos los cooperativistas en cuanto a la disciplina para alcanzar las normas de calidad y el volumen de producción prefijados, en el segundo.

Algunas de estas cooperativas se sitúan con índices de rentabilidad que les permiten reinvertir parte de sus ganancias en la modernización de sus infraestructuras y la consecución de otras iniciativas paralelas a la producción como la investigación de técnicas y 
manejos del suelo, la promoción de sus artículos en ferias, o la formación de sus socios por especialistas. En algunos de estos casos en que los agricultores de toda una comarca unen sus esfuerzos bajo formas democráticas de economía social y unos mismos modelos de producción agroecológicos, en torno a los cuales surge un proceso de identificación colectiva, algunos antropólogos trabajan sobre la hipótesis de si se desarrolla una auténtica cultura cooperativa ecológica (Del Campo, 1998; Del Campo, 2000a, Del Campo, 2000b). Para la antropología social, un determinado modo de producción en un contexto histórico y social específico puede generar identidades sociales que aglutinen a un colectivo humano en torno a su cultura del trabajo. La eclosión de un sistema de valores compartidos y unos esquemas mentales con los que interpretar la realidad cotidiana se origina en el quehacer diario de unos individuos que comparten un mismo nicho ecológico, un modo de producción que conserva esos recursos naturales, unas condiciones de trabajo comunes y unos objetivos económicos y socioculturales compartidos.

\section{Estudio de caso: La Cooperativa Olivarera Los Pedroches}

La comarca del Valle de los Pedroches ${ }^{10}$ es una extensa área de $3.613 \mathrm{~km}^{2}$, situada al norte de la provincia de Córdoba sobre un batolito granítico, vasta planicie cuajada de encinas formando un paisaje adehesado de un gran valor medioambiental, atravesado por numerosos filones de cuarzo y pórfido que originan las ondulaciones del terreno. La Cordillera Bética y la Sierra Morena han mantenido a esta zona durante siglos en semiaislamiento, hasta que en los años 70 y 80 mejoraron las vías de comunicación. A pesar de la extensión de la comarca, sus 17 municipios sólo albergan al 13\% de la población cordobesa, excluyendo la capital, unas 59.300 personas. Más de la mitad de la población se concentra en tres núcleos principales: Pozoblanco, Villanueva de Córdoba e Hinojosa. La despoblación de la zona (sólo 16 habitantes $/ \mathrm{km}^{2}$ ) es casi el doble de la media provincial, especialmente en los espacios rurales. La evolución de la población ha sido decreciente desde comienzos de la década de los cincuenta y hoy la estructura poblacional presenta síntomas de envejecimiento, así como de estrangulamiento en el segmento poblacional en torno a los 45 años, fruto de la emigración de décadas pasadas. Esta comarca se corresponde a un paisaje rural caracterizado por un secano cerealista y pradizales extensivos (Molinero y Alario, 1994), con un alto grado de especialización agraria extensiva y tecnificada, pero con graves defectos estructurales en la dimensión de las parcelas y explotaciones.

En cuanto al nivel de formación de la población, las últimas estadísticas (Instituto de Estadística de Andalucía, 1991) revelan que casi la mitad de los 60.000 habitantes de la comarca es analfabeta o no posee ningún estudio reglado, mientras que tan sólo el 3,4\% tiene o cursa actualmente estudios superiores. Además, dado que la estructura de la población está muy envejecida y que cuenta con gran cantidad de mujeres, que desempeñan su actividad en el hogar, el porcentaje de población activa es inferior al $60 \%$, ocupándose mayoritariamente en el sector primario $(39,2 \%)$, seguido del sector servicios $(30,3 \%)$. Resulta significativo que en 11 de los 17 municipios la población ocupada en el sector pri-

10 Los Pedroches está integrado por los siguientes 17 municipios: Añora, Belalcázar, Dos Torres, Pozoblanco, Pedroche, Villanueva de Córdoba, Fuente La Lancha, Alcaracejos, Santa Eufemia, El Guijo, Torrecampo, Conquista, Hinojosa del Duque, El Viso, Cardeña, Villanueva del Duque y Villaralto. Todos ellos constituyen desde 1993 la Mancomunidad de municipios de Los Pedroches. 
mario sea superior al $40 \%$. En cuanto a la renta disponible, ésta se situaba a principios de los 90 en 507.000 ptas., cifra inferior a la media provincial (631.897 ptas.).

Frente a esta desalentadora situación socioeconómica, se ha constatado recientemente la consolidación de un asociacionismo empresarial que venía gestándose desde los años sesenta, centrado en favorecer las primeras transformaciones de los productos de la comarca y que algunos califican hoy de «ejemplar para una zona con los recursos y problemas de Los Pedroches» (Ramos, 1997). Asimismo se han asentado también en la última década numerosas organizaciones no institucionales dedicadas a la defensa y el desarrollo de la comarca (Asociación para el Desarrollo de Los Pedroches, Mancomunidad de Los Pedroches, Grupo de Acción Local, Proyecto Los Pedroches S.A., etc.). Desde estas agrupaciones se perfilan diferentes estrategias de desarrollo comarcal. Una de las más importantes pasa por la potenciación de los sistemas agrarios respetuosos con el medioambiente, en buena medida también impulsado por las iniciativas europeas a través del Programa Medioambiental 2000/06 y de los programas LEADER y PRODER. Este contexto de cooperación y asociacionismo ha impregnado con mayor fuerza que ninguna otra actividad al sector agroalimentario. Tras el sector cárnico en dehesas y los cultivos herbáceos, el olivar ha sido tradicionalmente el eje económico del sector primario, especialmente en la localidad más grande, Pozoblanco, donde se encuentran extensas manchas de olivares cultivados tradicionalmente sobre pendientes escarpadas.

Este cultivo es precisamente la razón de ser de una de las múltiples cooperativas ubicadas en esta comarca: la Cooperativa Olivarera Los Pedroches, fundada en 1957, con sede en Pozoblanco. De sus más de 500 socios, la mayoría ha convertido progresivamente sus tierras a cultivos ecológicos, con un crecimiento continuo desde los inicios de la campaña 1994/1995, cuando el éxito de los primeros 14 agricultores animó a 191 olivareros más. En la actualidad son ya más de 400 los cooperativistas que cultivan con métodos ecológicos una superficie total de unas 7.000 has., produciendo más de $1.000 \mathrm{Tm}$. de aceite anuales que son almacenadas en bodegas capaces de albergar hasta 1.400. Bajo una forma de economía social y cogestión empresarial, este colectivo ha ido aunando esfuerzos hasta consolidarse hoy como el principal núcleo de producción ecológica en Andalucía y uno de los más relevantes del país.

Para que este colectivo compita hoy en prestigio y calidad con las más reputadas marcas de aceite se han necesitado la voluntad y el esfuerzo conjunto de todos sus socios. Y el esfuerzo empieza en el campo. Las pronunciadas pendientes de gran parte de estos olivares han dificultado siempre el laboreo y la recolección. El tractor es una herramienta inútil en muchas fincas de la sierra. Hay que trabajar a mano, en ocasiones atado al árbol para no despeñarse. Para mayor dificultad, el abuso de agroquímicos, el laboreo intensivo, la eliminación del manto vegetal y otras prácticas abusivas, hicieron peligrar el ecosistema y lo empobrecieron aún más. Desaparecida la vegetación que fijaba la tierra al suelo, las escorrentías hicieron el resto y erosionaron el campo hasta niveles insostenibles. El suelo llegó a perder 80.000 kilos de tierra por hectárea. Los olivos cada vez producían menos en comparación con las campiñas de Jaén, Sevilla y el sur de Córdoba. Los olivareros se concienciaron: había que renovarse o morir. Así que acogieron la opción ecológica con entusiasmo, invirtieron para mejorar tecnológicamente su producción sin desechar las soluciones tradicionales y a mediados de los 90 fueron capaces de elaborar un aceite ecológico de intenso sabor a fruta madura. No escatimaron en medios tecnológicos. Sólo para controlar la mosca del olivo, por poner un ejemplo, experimentaron con tratamientos aéreos con piretrinas naturales, rotenona y atrayente sexual; trampeo masivo con botellas y atracción alimenti- 
cia de fosfato; trampas lona, trampas de cartón, etc. Alentados por la mejora de sus campos, el trabajo en un entorno laboral más gratificante, la satisfacción de producir unos alimentos de la máxima calidad y la rentabilidad económica de la iniciativa, decidieron en 1998 comercializar su aceite con su propia marca: Olivalle, un jugo muy afrutado obtenido mediante presión en frío de aceitunas seleccionadas de la variedad Nevado Blanco. Hoy contemplan su obra con satisfacción y optimismo.

Una actividad socioeconómica alternativa (la industria olivarera ecológica), gestionada con modelos de producción, gestión y organización empresarial no convencionales (cooperativas) en un marco ecológico y sociocultural común (ecosistema olivar) y una experiencia histórica básicamente compartida (subdesarrollo comarcal, estructura de pequeña propiedad, dificultades por la baja productividad de los olivos, etc.) está generando unos determinados procesos de identificación colectiva que superan el mero asociacionismo económico. Agricultores y no agricultores, socios y colaboradores de la cooperativa, pequeños y grandes propietarios, titulares o arrendatarios de las explotaciones, construyen día a día una comunidad de valores compartidos centrada en una cosmovisión indisociable de su modo de producción. No se trata sólo de lo que los sociólogos llaman roles, pautas de comportamiento definidas por normas estructuradas por las instituciones y organizaciones de la sociedad. Estamos ante un ejemplo de vigorosa expresión de una identidad colectiva comarcal, fuente de sentido construida en el día a día, que desafía la globalización y el cosmopolitismo en nombre de la singularidad cultural y el control de la gente sobre sus vidas y entornos (Castells, 1998; Del Campo 2000a).

Iniciativas de desarrollo económico local de carácter endógeno como ésta, crean empleo, diversifican la producción, dinamizan la comarca y construyen además un universo de percepciones, valores y sentimientos que confiere a sus actores una urdimbre de significados (Geertz, 1997) con la que interpretar su realidad cotidiana. Ante la insostenibilidad de un modelo de producción que enajenaba lentamente los recursos naturales de la comarca, los cooperativistas de Los Pedroches han sabido adaptarse a los nuevos condicionantes con esfuerzo, imaginación y compromiso. En términos de Bennett, el profundo cambio social experimentado por este colectivo puede ser definido como proceso adaptativo consistente en una estrategia colectiva, elegida libremente, aplicada durante un período de tiempo considerable y dirigida a obtener y usar los recursos para resolver los problemas inmediatos del colectivo. O si se prefiere en términos de Rappaport (1971), la iniciativa ecológica de la Cooperativa Olivarera Los Pedroches supone un «mecanismo regulador del sistema» en cuanto que «en respuesta a cambios duraderos en el entorno cambian su organización, su estructura y su funcionamiento» (Rappaport, 1971).

Si como dicen los filósofos Jorge Riechmann y Francisco Fernández Buey (1994), «la dinámica de las modernas sociedades industriales ha puesto en marcha un proceso probablemente irreversible de disolución de los vínculos sociales y las identidades tradicionales», iniciativas socioeconómicas como ésta reagrupan y construyen procesos de identificación colectivos que reintegran al individuo a su identidad territorial. Con su trabajo diario, estos hombres y mujeres están provocando el desmoronamiento de la imagen tópica de Los Pedroches como un valle subdesarrollado y olvidado. Hoy, el diagnóstico del sector agroalimenario en la comarca es profundamente alentador: «la especial relevancia de la industria agroalimentaria en la comarca destaca por su tradición, por su responsabilidad en la mayor parte de las innovaciones que se han llevado a cabo en los últimos años, por su identificación con la imagen comarcal y por la potencialidad de este sector, derivada del alto nivel de calidad de los productos de la zona» (Ramos, 1997). 


\subsection{La viabilidad socioeconómica de la agricultura ecológica a la luz de la experiencia de Los Pedroches}

Algunos autores piensan que «la creencia de que la agricultura ecológica no es viable desde el punto de vista económico ha sido uno de los factores principales que ha limitado en el pasado la expansión de dicho sistema agrario» (Lampkin, 1998). El caso de Los Pedroches es paradigmático, pues a pesar de la demostrada viabilidad socioeconómica del proyecto son aún muchos los que recelan de su futuro. Esta cooperativa no es en modo alguno un caso aislado. En el mayor estudio socioantropológico llevado a cabo en Andalucía (Del Campo, 2000a) se comprobó, tanto en grandes como en pequeños agricultores, la rentabilidad económica de unos agrosistemas que soportan un rendimiento productivo del $10 \%$ al $30 \%$ más bajos de promedio ${ }^{11}$, pero que logran equilibrar este déficit gracias fundamentalmente a las subvenciones públicas y los mayores precios obtenidos por sus productos ecológicos. Lamentablemente aún faltan en Andalucía estudios comparativos de rendimientos que analicen los márgenes de rentabilidad en los agros ecológicos y convencionales de unos mismos productos como los que se han llevado a cabo en países centroeuropeos y norteamericanos. En 1998, la revista Nature publicó las conclusiones de una investigación llevada a cabo en el Royal Institute de Pensilvania (EE.UU.), en la cual varios científicos derribaron uno de los mitos que sustenta la agricultura convencional; aquel según el cual la agricultura ecológica es simplemente menos eficaz y productiva que la manejada con abonos químicos de síntesis. Durante 15 años se cultivaron al lado de una explotación convencional, dos fincas en régimen ecológico. La media anual de cosechas fue similar en ambos casos (7.120 kilos de maíz ecológico por hectárea y 7.170 kilos de maíz convencional por hectárea). Los beneficios económicos no diferían significativamente, sin embargo, las investigaciones concluían que durante este período de tiempo se filtró un $60 \%$ más de nitrógeno en el cultivo convencional que en el ecológico. Este tipo de contaminación es el causante de que muchos de nuestros pozos (como el de Almodóvar del Río en Córdoba, por ejemplo) ya no sean potables desde hace años. El estudio demostraba que un uso ecológico prolongado genera sustanciales beneficios en cuanto al aumento de fertilidad, el respeto a la biodiversidad, y la ausencia de contaminación, mientras que la productividad y rentabilidad en ningún caso se veía dañada por la ausencia de productos químicos de síntesis (fertilizantes, plaguicidas, etc.).

A pesar de que muchos agricultores ecológicos andaluces manifiestan que su producción es superior al de sus homólogos convencionales, las investigaciones realizadas (Del Campo, 2000a) demuestran que no todos son capaces o tienen el tiempo de alcanzar los rendimientos máximos a los que suele llegar la agricultura química, especialmente en aquellos cultivos donde son necesarias medidas que merman la capacidad productiva inmediata, como por ejemplo, un aumento del espacio entre las hileras de vegetales para mantener a las malas hierbas bajo control. Las diferencias de rendimiento productivo varían en función de múltiples factores pero sí se ha observado en Andalucía que los suelos especialmente fértiles muestran índices de producción prácticamente equivalentes en cultivos ecológicos y convencionales. En algunos casos los agricultores de productos hortícolas y frutales especialmente, han comprobado tras varios años de producción ecológica que los rendimientos

11 Como por otra parte recogen la mayoría de los estudios extranjeros: p.ej. Vine y Bateman, 1981. 
superan ya a la época en que cultivaban con métodos convencionales. En otras zonas de baja productividad, como el caso analizado de la comarca del Valle de Los Pedroches, en Córdoba, sus olivos no pueden competir en cantidad con los de la campiña jienense o sevillana, pero sí en calidad, por lo que los bajos rendimientos se ven compensados por los altos precios que alcanza el aceite de oliva virgen ecológico.

Este sobreprecio, que oscila entre un 20 y un $80 \%$ más con respecto a los productos convencionales en el mercado nacional, supone, junto a la creciente demanda externa, principalmente de los mercados centroeuropeos y más recientemente asiáticos, donde se alcanzan unos sobreprecios de hasta el $300 \%$, uno de los factores principales de viabilidad económica para la agricultura ecológica andaluza. Por otra parte, los aumentos de coste en semillas, tiempo de trabajo y abonos orgánicos, se ven compensados por costes mucho menores en fertilizantes y plaguicidas, especialmente a medio y largo plazo cuando las plagas se equilibran y el rendimiento del suelo ha aumentado. Finalmente las ayudas públicas a la reconversión han supuesto, especialmente en los tres últimos años, un sabroso incentivo para muchos agricultores, particularmente para propietarios de fincas de bajo rendimiento. El objetivo de las subvenciones radica en compensar las pérdidas que el agricultor experimenta tras los primeros años de reconversión. En este sentido algunos agricultores ecológicos - fundamentalmente propietarios de grandes explotaciones de olivos- muestran después de cuatro años tal superávit que su rentabilidad está asegurada incluso si desaparecieran las subvenciones de la Administración. Otros, por el contrario - pequeños horticultores-, permanecen en el mercado gracias a dichas ayudas y su futuro es incierto en caso de que estas desaparezcan. Igualmente se ha observado en el curso de la investigación que existen algunos agricultores cuya única cuita parecer ser la consecución de la mayor subvención posible de manera que obtengan unos ingresos aun sin explotar la tierra o que reconvierten una superficie mínima de su producción total para utilizar después la imagen ecológica en hábiles estrategias comerciales.

Es difícil saber con precisión el margen de maniobra que tendría la agriculura ecológica andaluza sin estas ayudas públicas. A la carencia de estudios comparativos concretos se le une otra dificultad: la enorme heterogeneidad de los propios agricultores ecológicos. Así existen multitud de explotaciones que han optado por la producción ecológica por motivos puramente crematísticos, mientras otros ven en la agricultura ecológica una terapia para la totalidad de las enfermedades del globo (económicas, socioculturales y medioambientales). Hay una agricultura ecológica muy productiva e intensiva, que aporta grandes dosis de fertilizantes al campo y que acostumbra a luchar contra las plagas y enfermedades con productos fitosanitarios comerciales. Hay otra que se contenta con unos volúmenes de producción menores pero un óptimo manejo ecológico que garantiza la sustentabilidad a largo plazo, y una tercera cuya experiencia agroecológica le permite desarrollar modelos productivos tan ecológicos como rentables, sin llegar a depender de las subvenciones públicas. Cultivos y fincas muy modernizados se contraponen a otros claramente marginales. El peso económico y la autonomía de los primeros contrasta con la extensividad superficial y la dependencia de las ayudas públicas de los segundos. Encontramos agricultores que en poco o nada han modificado su hábitos productivos, bien porque ya practicaban antes una agricultura tradicional, bien porque se limitan ahora a sustituir los productos químicos de síntesis por otros biológicos, mientras que otros representan una auténtica ruptura con la agricultura convencional, y establecen una verdadera cultura ecológica que impregna todos los ámbitos de la vida. Existen agricultores que optan por la producción ecológica porque sus padres y sus abuelos mantenían un cultivo tradicional, e inversamente, hay agricultores 
que han visto en la agricultura ecológica una vía para modernizarse y diferenciar su producción, así como para experimentar nuevas técnicas alternativas. En cualquier caso, y en espera de estudios exhaustivos que arrojen datos más exactos, podemos concluir que los beneficios económicos finales de los agricultores ecológicos andaluces se mueven en índices equivalentes e incluso, en algunos casos, superiores a las producciones convencionales, de tal manera que conocemos muchas explotaciones cuya viabilidad no depende de las ayudas públicas.

No obstante el cálculo de costes y beneficios económicos no refleja toda la problemática ecológica de la agricultura. Los sistemas agrarios convencionales conllevan costes para la sociedad que son más dificiles de cuantificar monetariamente pero que repercuten sin duda en las arcas del presupuesto comunitario, estatal y autonómico. La reparación de los desastres ambientales, las costosas depuradoras que logran suministrar agua limpia de nitratos, el control de los residuos de plaguicidas en nuestros alimentos, la conservación y mejora de paisajes especialmente dañados, son sólo algunos de los costes sociales que debemos pagar por el actual modelo agrario (Altieri, 1993; Lampkin, 1998). Un modelo agrónomo como el ecológico, que reduce estos costes externos y permite una rentabilidad económica ${ }^{12}$ y social para nuestros agricultores, puede ser una eficaz alternativa para el campo andaluz.

\section{Reflexiones finales}

Mundialmente existe el consenso de que la agricultura del tercer milenio necesita nuevas estrategias de desarrollo que aseguren una producción estable de alimentos sin poner en peligro los recursos naturales para las futuras generaciones, puesto que «no es seguro que las agriculturas más avanzadas, grandes consumidoras de energía, tengan ante ellas un porvenir demasiado duradero», como vaticina el antropólogo Pierre Bonte (Bonte e Izard, 1996). La agricultura ecológica puede ser propuesta como un sistema productivo alternativo que frene los devastadores efectos medioambientales provocados por la agricultura convencional: alto coste energético, reducción gradual del contenido de materia orgánica edáfica, empobrecimiento del patrimonio genético autóctono, contaminación de aguas superficiales y subterráneas, salinización de acuíferos, eutrofización de aguas continentales, pérdida de calidad organoléptica de los alimentos, etc.

Pero además de las ventajas medioambientales, se aprecian sobre todo las territoriales ya que la agricultura ecológica puede constituir una estrategia efectiva de desarrollo que contribuya a paliar la degradación y desarticulación del agrosistema rural desde un punto de vista económico y sociocultural. Nos referimos al éxodo rural, la desocialización del agricultor, la pérdida de relación con la tierra, la progresiva dependencia con sistemas productivos externos, y otros factores que deben hacernos reflexionar - a científicos, productores y miembros de la Administración-, sobre la idoneidad de continuar con nuestro actual sistema productivo agrario. Si el agricultor en la sociedad industrial, como ha dicho E. Fromm, «debe estar abierto a las nuevas ideas, ser cooperativo en cierta medida, capaz de planear y de invertir para el futuro y estar dispuesto a involucrarse en nuevas iniciativas de desarrollo que rompan con su tradicional modo de producción» (Fromm et al., 1973), la agricultura

12 La Unión Europea considera la importancia de los métodos de producción ecológica en relación a sus importantes beneficios medioambientales y al aumento del trabajo agrícola (con el consiguiente incremento del empleo). 
ecológica parece representar en muchos contextos no sólo un eficaz instrumento de desarrollo económico y articulación territorial sino un elemento cohesionador de determinadas identidades colectivas que se aglutinan en torno a este sistema de producción alternativo.

Sin embargo a nadie se le escapa las dificultades que entraña un nuevo modelo de producción. La raquítica demanda de los consumidores españoles, la carencia de ejes comerciales que articulen a los distintos productores, y, como consecuencia, los elevados precios, hacen que la agricultura ecológica en España dependa de factores exógenos (demanda de los países centroeuropeos, competencia con otros productores del mediterráneo como Argelia, etc.). A pesar de éstos obstáculos, algunos agricultores como los cooperativistas de Los Pedroches, aquí descritos, nos alejan del estereotipo utópico y romántico con que hasta hace pocos años se les identificaba. Rentabilidad económica, ética laboral y respeto al medioambiente dejan de ser en estos casos ideales imposibles de cumplir conjuntamente. De que seamos capaces o no de consolidar iniciativas como ésta, dependerá que el cuestionamiento del actual sistema productivo no sea una mera reflexión metafísica.

\section{Bibliografía}

ALONSO, A.M. y GUZMÁN, G.I. (1998): Cultivo del olivar en agricultura ecológica. Plan de Formación CAAE., boletín no 3.2. Sevilla.

AURIOLES, J. et al. (1980): Cuentas económicas de Andalucía. Tablas Input-Output. Junta de Andalucía.

BONTE, P. e IZARD, M. (1996): «Agricultura» en Diccionario de Etnología y Antropología. Akal. Madrid.

CALERO, L. (1999): «Ensayos en la comarca de Los Pedroches para el control de bactrocera oleae (dacus o.): con técnicas de agricultura ecológica» en Humus, revista de agricultura ecológica, $\mathrm{n}^{\circ}$ 2, febrero.

CÁNOVAS, A. et. al. (1993): Tratado de Agricultura Ecológica. Instituto de Estudios Almerienses. Diputación Provincial de Almería.

CASTELLS, M. (1998): La era de la información. Economía, Sociedad y Cultura. Vol. 2.: El Poder de la Identidad. Alianza. Madrid.

CÉNIT, M., ALONSO, A.M., GUZMÁN, F. (1996): Estudio de Mercado para productos ecológicos: Estudio del segmento de demanda de los centros permanentes de consumo en la provincia de Málaga. Proyecto mixto de colaboración entre la Consejería de Agricultura y Pesca y UAGA-COAG.

CEPA (1993): Estudio exploratorio del consumo de productos ecológicos de la agricultura ecológica en la provincia de Sevilla. Confederación Ecologista y Pacifista Andaluza y Asociación de Productores y Consumidores La Ortiga. Sevilla.

COLMENARES, R.; PÉREZ-SARMENTERO, J.; MOLINA, A. (1994): «La agricultura ecológica: construyendo la agricultura del mañana» en Agricultura y medio ambiente. El Campo, Servicios de Estudio BBV.

DEL CAMPO, A. (1998): Cultura cooperativa. Desarrollo sostenible y articulación territorial. Las cooperativas agropecuarias de la comarca del Valle de los Pedroches (Córdoba). Proyecto de Investigación. Departamento de Antropología Social, Universidad de Sevilla (inédito).

DEL CAMPO, A. (2000a): Agricultores y Ganaderos Ecológicos en Andalucía. Despegue, Consolidación y Futuro de un sistema de producción y un modelo de desarrollo alternativo. Consejería de Agricultura y Pesca, Junta de Andalucía. 
DEL CAMPO, A. (2000b): Seis años de agricultura y ganadería ecológica en Andalucía. Crónica de la consolidación del modelo productivo ecológico. Edit C.A.A.E., Sevilla.

DOXA, S.A. (1991): Estudio sobre el mercado de la agricultura ecológica. MAPA-INDO. Madrid.

ECOMARKET (1993): Actitud de los consumidores respecto a los alimentos ecológicos. Comentarios a un sondeo piloto en la zona metropolitana de Granada (Documento de Trabajo). Departamento de Economía y Sociología agrarias. C.I.D.A. Granada. Dirección General de Investigación, Tecnología y Formación agroalimentaria y pesquera, Consejería de Agricultura y Pesca, Junta de Andalucía.

FROMM, E. y MAC COBY M. (1973): Sociopsicoanálisis del campesino mexicano. Estudio de la economía y la psicología de una comunidad rural. Fondo de Cultura Económica. México.

GIVORD, D. (2000): «Defensa del modelo rural y agrario europeo en la OMC», en revista LEADER Magazine $n^{\circ} 25$.

GUTIÉRREZ, F.; ARNAUD, T. y ALBI, M.A. (1996): Influencia del cultivo ecológico del olivar sobre la calidad del aceite de oliva virgen obtenido. Instituto de la Grasa, Consejo Superior de Investigaciones Científicas (C.S.I.C.) Sevilla.

GUZMÁN, G.I. (1995): «Estilos de agricultura ecológica. La agricultura ecológica en Andalucía» en Agroecología y Conocimiento Local, Programa de Doctorado: Agroecología, Campesinado e Historia. Universidad Internacional de Andalucía. Instituto de Sociología y Estudios Campesinos y E.T.S.I.A.M. de la Universidad de Córdoba. Santa María de la Rábida.

HOLSTROM, M. (1994): The New Spanish Social Economy. Berg Publishers. Londres.

LABRADOR, J. y GUIBERTEAU, A. (1990): La Agricultura Ecológica. Hojas Divulgativas, $n^{\circ} 11 / 90$ HD. Secretaría General de Estructuras Agrarias. Ministerio de Agricultura, Pesca y Alimentación.

LAMPKIN, N.H. (1998): Agricultura ecológica. Edicionea Mundi-Prensa. Madrid.

LOWE, P., MURDOCH, J. y WARD, N. (1997): «Redes en el desarrollo rural: más allá de los modelos exógenos y endógenos» en Rev. Agricultura y Sociedad no 82 (EneroAbril).

MOLINERO HERNANDO, F. y ALARIO TRIGUEROS, M. (1994): «La dimensión geográfica del desarrollo rural: Una perspectiva histórica» en Revista de Estudios Agrosociales. Núm 169 (julio-septiembre).

NAVARRO LUNA, J. y DEL CAMPO, A. (1999): Agricultura y ganadería ecológica en Andalucía. Las Regiones Españolas en Europa, Actas de la XXV Reunión de Estudios Regionales. Sevilla.

NAVARRO LUNA, J. y VENTURA FERNÁNDEZ, J. (1999): «Mancomunidades, consorcios y comarcas de desarrollo rural en Andalucía» en Revista El siglo que viene ${ }^{\circ}$ 38. Sevilla.

RAAE. (Red Andaluza de Experimentación Agraria) (1994): Caracterización de la Agricultura Ecológica en Andalucía. Dirección General de Investigación Agraria. Sevilla.

RAMOS, E. (1997): Programa de Innovación Rural de Los Pedroches. Un modelo de diagnóstico y formulación participativos. Grupo de Acción Local Proyecto Los Pedroches, S.A. Publicaciones Obra Social y Cultural Cajasur. Córdoba.

RAPPAPORT, R. (1971): «Nature, Culture and Ecological Anthropology» en Man, Culture and Society. Shapiro, H. (ed.): Oxford University Press. Oxford. 
REIJNTES, C.; HAVERKORT, B.; WALERS-BAYER, A. (1992): Farming for the future. An introduction to Low-External-Input and Sustainable Agriculture. Ed. MacMillan Press. Londres.

RIECHMANN, J. y FERNÁNDEZ-BUEY, F. (1994): Redes que dan libertad, Introducción a los nuevos movimientos sociales. Ediciones Paidós. Barcelona.

ROHRSCHNEIDER, R. (1988): «Citizen's Attitude toward Environmental Issues. Selfish or Selfness?» en Comparative Political Studies, vol. 21, $\mathrm{n}^{\circ} 3$.

SEVILLA, E. (1992): «Una propuesta de desarrollo rural endógeno para Andalucía» en Revista de Estudios Regionales, $\mathrm{n}^{\circ} 31$.

SIMÓN FERNÁNDEZ, X. (1995): «Economía ecológica, agroecología y desarrollo rural sostenible» en Rev. Agricultura y Sociedad no 77 (Octubre-Diciembre).

SUMPSI VIÑAS, J.M. (1994): «La Política agraria y el futuro del mundo rural» en Rev. De Estudios Agro-Sociales no 169 (julio-septiembre):.

TOLEDO, V. (1992): «Regresemos al agro» en Cuadernos Verdes, Colegio Verde de Villa de Leyra, $n^{\circ} 5$.

VALLE BUENESTADO, B. (1985): Geografía agraria de Los Pedroches. Córdoba.

VV.AA. (2000): Hechos y Cifras. Ministerio de Agricultura, Pesca y Alimentación. Secretaría General Técnica. Madrid, pp. 103. 